\title{
Blood pressure management in the elderly: the need for more randomised evidence
}

\author{
Ana-Catarina Pinho-Gomes ${ }^{1,2}$, Kazem Rahimi ${ }^{1,2,3 *}$
}

1. Deep Medicine, Oxford Martin School, Oxford UK

2. George Institute for Global Health, University of Oxford, Oxford, UK

3. National Institute for Health Research Oxford Biomedical Research Centre, University of Oxford, Oxford, United Kingdom

*kazem.rahimi@georgeinstitute.ox.ac.uk

Our understanding of relationships between blood pressure (BP) and major vascular outcomes has greatly advanced. Yet there remain important uncertainties which affect guideline recommendations and treatment decisions. One major area of uncertainty and controversy is treatment of BP in elderly patients, in particular when BP values are not very high.

In this issue of the Heart, Jung et $\mathrm{al}^{1}$ aim to investigate this question further. Using the Korean National Health Insurance data, they identify a cohort of about 420,000 individuals who are followed up for about 10 years to investigate adjusted associations for death due to cardiovascular disease (CVD). Overall, the study confirms the growing evidence on the graded association between BP and risk of CVD. The authors report a $40 \%$ increased risk of CVD death per $20 \mathrm{mmHg}$ higher baseline systolic BP, with no evidence of a change in pattern at any particular BP threshold. Also in keeping with previous literature, the strength of the association with BP is shown to vary depending on the specific type of CVD outcome, with a weaker association observed for ischaemic heart disease (IHD) than stroke, particularly haemorrhagic stroke. The study also adds to the existing literature by implicitly showing no evidence of major difference in patterns of associations by ethnicity.

But, how does this study contribute to the question of BP management in elderly patients? The investigators divide the population into three age groups of 40-59, 60-69, and 70-80 years and repeat analysis for each group separately. Again, consistent with the previous literature, they observe a declining slope of association with increasing age. Even in the oldest patient group, there was a log-linear association between BP and risk of CVD death; for each $20 \mathrm{mmHg}$ higher systolic BP at baseline, the risk of CVD death during follow-up was $22 \%$ higher (confidence interval 16 to $29 \%$ ). When total CVD deaths were divided into stroke (ischaemic and haemorrhagic) and IHD, a similar pattern was observed, however, with the exception of IHD, where no statistically significant graded positive association was discernible. This latter finding from the subgroup analysis led to the conclusion that "An individualized approach considering each patient's personal risk profile is needed when initiating BP interventions for the elderly".

In our view, this statement might be an overinterpretation of the results and deserves further scrutiny. A closer look at their results (see Figure 2) shows that there is nothing particularly special about the associations for IHD death in the elderly group. With 'only' 23,451 participants in the oldest group, and given the well-known weaker association between BP and IHD (compared with stroke), it is plausible to assume that the apparent differences are due to the study's limited statistical power. The authors also used a single measure of BP at baseline which is prone to measurement error and variability and hence could have further diluted associations. Evidence has been mounting on the substantial variation of BP over time, raising questions about the accuracy of isolated 'baseline' BP values to estimate CVD risk. In particular, among elderly and multimorbid patients, the value of repeated measurements for safety monitoring and treatment adjustment are likely to be of particular importance, given their altered drug metabolism and increased vulnerability 
to adverse events. Another potential issue of the analysis, similar to many other observational studies, is the greater challenge of mitigating reverse causality in elderly patients. Such patients are more likely to have several known or incipient conditions that could bias risk estimates. The authors report that they excluded patients who died within the first three years to tackle this issue but the extent to which this would overcome the challenge is unclear. On the one hand, as a previous study that investigated the risk of BP on vascular dementia has reported, the exclusion of just three years of follow-up might not be sufficient to unmask reverse causation. ${ }^{2}$ On the other hand, exclusion of additional years of follow-up would inevitably reduce statistical power. Finally, even if the observed associations were causal, it is not clear how clinicians could individualise BP management, given that decisions to treat are usually not based on a single component of cardiovascular disease, such as IHD. Indeed, one could argue that given that among elderly patients, the risk of stroke and heart failure are particularly high, a lack of a graded association for IHD death would not add much weight to clinical decision making.

Where does this study leave us in terms of the key question about treatment of BP in elderly? In our view, the answer is unlikely to come from further non-interventional studies. This is not only due to inherent limitations of observational studies to establish causation but also because they tend to exclude people with known disease at baseline. Although this design feature is appropriate from a methodological perspective, it renders study findings less relevant to our contemporary elderly population. Indeed, diseases tend to accrue with age, which underpins the rapid increase in multimorbidity secondary to population ageing. The group of elderly individuals with multimorbidity and frailty is the one in whom BP management remains more controversial as they have been typically excluded by clinical studies.

Unfortunately, this issue is not limited to observational studies. A recent systematic review that examined phase III randomised controlled trials funded by the National Institutes of Health from 1965 to 2015 concluded that "beyond explicit exclusion by age, older adults were often implicitly excluded based on various comorbid conditions such as polypharmacy/concomitant medication (37\%) or cardiac issues (30\%)". ${ }^{3}$ The same issue applies to several trials of BP lowering treatment. Although individual clinical trials and meta-analysis of these have shown no evidence of a significant difference in treatment effects in younger ( $<65$ years) vs older ( $\geq 65$ years) patients, the age range included in these studies has been narrow. ${ }^{4}$ More importantly, those studies had limited information on tolerability and safety outcomes, which are common reasons for withholding treatment in elderly and multimorbid patients. This lack of evidence is acknowledged by the most recent hypertension guidelines from the National Institute for Health and Care Excellence in the UK, which recommended using clinical judgement when making treatment decisions in elderly people with frailty and multimorbidity. ${ }^{5}$ International guidelines and professional societies likewise summarise the evidence and come to the conclusion that more research is needed in the very elderly and frail. ${ }^{6}$

There is thus a pressing need for more reliable evidence to thoroughly address this area of unmet clinical need. In the first instance, this could be achieved by pooling evidence from randomised clinical trials that are currently available. The Blood Pressure Lowering Treatment Trialists' Collaboration has recently been extended to now include the largest source of individual participantparticipant data from randomised controlled trials. ${ }^{7}$ The gathered information from over 350,000 randomised patients includes over 20,000 patients who were over 80 years at the time of randomisation. Thus, this dataset provides a unique opportunity to investigate key questions about overall effects of BP-lowering treatment on cardiovascular outcomes by age and morbidity status. Nonetheless, there are questions that even such unprecedented analysis might not be able to address. For instance, recent guidelines suggested that harms may outweigh benefits in the elderly or those with multimorbidity, due to higher rates of adverse events and/or because the expected benefit from continuing a preventive medicine is reduced when there is limited life expectancy or 
high risk of death from competing diseases" ${ }^{8}$ Trials are currently underway or in planning stage to investigate the effect of intensive BP lowering on patient-important outcomes in elderly multimorbid patients, which will hopefully resolve some of most critical issues pertaining to this growing patient population.

In conclusion, the population-based study by Jung et al. ${ }^{1}$ provides reassurance about an overall positive association between BP and CVD risk in the elderly in a non-Western population. It further highlights the need for more reliable evidence from randomised trials to assess the balance of safety and efficacy of antihypertensive treatment in elderly patients, in particular when BP is not very high and in presence of comorbidities. Until such evidence becomes available, 'clinical judgement' with its inherent limitations will remain the best standard that guidelines can recommend. 


\section{References}

1. Jung M-H, Yi S-W, An SJ, Yi J-J. Age-specific associations between systolic blood pressure and cardiovascular mortality. Heart 2019.

2. Emdin CA, Anderson SG, Salimi-Khorshidi G, et al. Usual blood pressure, atrial fibrillation and vascular risk: evidence from 4.3 million adults. International journal of epidemiology 2017; 46(1): 162-72.

3. Lockett J, Sauma S, Radziszewska B, Bernard MA. Adequacy of Inclusion of Older Adults in NIH-Funded Phase III Clinical Trials. J Am Geriatr Soc 2019; 67(2): 218-22.

4. Turnbull F, Neal B, Ninomiya T, et al. Effects of different regimens to lower blood pressure on major cardiovascular events in older and younger adults: meta-analysis of randomised trials. Bmj 2008; 336(7653): 1121-3.

5. NICE. Hypertension in adults: diagnosis and management (draft for consultation). London, UK: National Institute for Health and Care Excellence, 2019.

6. Williams B, Mancia G, Spiering W, et al. 2018 ESC/ESH Guidelines for the management of arterial hypertension. European Heart Journal 2018; 39(33): 3021-104. 7. Rahimi K, Canoy D, Nazarzadeh $\mathrm{M}$, et al. Investigating the stratified efficacy and safety of pharmacological blood pressure lowering: An overall protocol for individual patient-level data meta-analyses of over 300,000 randomized participants in the new phase of the Blood Pressure Lowering Treatment Trialists' Collaboration (BPLTTC). BMJ Open 2019. 8. NICE. Multimorbidity: the assessment, prioritisation and management of care for people with commonly occurring multimorbidity. NICE guideline [NG56]. UK: National Institute for Health and Clinical Excellence; 2016. 